\title{
DIE VERTEILUNG DER BIENEN IM STOCK NACH ALTERSKLASSEN WÄHREND DER WANDERUNG
}

\author{
Répartition des abeilles dans la ruche selon les \\ classes d'âge au cours de la transhumance
}

\author{
Zoltán ÖRÖSI-PÁL
}

Abteilung Bienenzucht, Forschungsanstalt für

Kleintierzucht, Gödöllö, Ungarn

\author{
SUMMARY \\ THE DISTRIBUTION AFTER AGE GROUPS OF THE BEES \\ IN THEIR HIVE DURING MIGRATION
}

\begin{abstract}
During migration the bees are neglecting brood rearing. While young bees are nurses, old bees are working as foragers. By marked bees it was investigated where worker bees of different ages are staying in the hive in transit. Young bees (1-17 days old) are keeping more quiet and are less active. Nevertheless, due to the shaking, a high number of worker bees left the brood. $30-56-70,8 \%$ of them were found in the upper (drum chamber )(Trommelraum), an empty, ventilated part of the hive. These facts give an explanation of the deficient brood rearing. At the end of the migration, the bees in the " drum chamber " weighed less than at the beginning, because they had no occasion to fill their honey stomachs.
\end{abstract}

\section{ZUSAMMENFASSUNG}

Die Bienen vernachlässigen die Brutpflege während der Wanderung. Junge Bienen sind Pfleger, alte aber Sammler. Mit gezeichneten Bienen wurde untersucht, wo sich Arbeiterinnen verschiedenen Alters in der transportierten Bienenwohnung aufhalten. Junge (1-17 Tage alte) Bienen sind ruhiger und weniger beweglich. Dennoch verließen sie infolge der Erschütterung die Brut in großer Zahl und wurden zu 30-56-70,8\% im oberen Trommelraum, einem leeren, belüfteten Teil des Stockes, vorgefunden. Die mangelhafte Brutpflege ist somit erklärt. Im Trommelraum war das Gewicht der Bienen am Ende der Wanderung geringer, weil sie dort keine Gelegenheit hatten, ihre Honigblasen zu füllen. 


\section{EINLEITUNG}

Man wandert mit den Bienenvölkern, um Bienenweiden aufzusuchen oder um die Bienen vor der Gefahr durch Chemikalien zu schützen.

Die Wanderung widerspricht der Natur des Bienenvolkes und kann sogar das Leben der Bienen und der Brut aufs Spiel setzen. Um diese Gefahr zu vermindern, muß man in der Bienenwohnung für leere Räume (“ Trommelräume ») und für reichliche Lüftung durch “ Wandergitter » sorgen.

Das Verhalten der Bienenvölker während der Wanderung habe ich mit Hilfe von elektrischen Widerstandsthermometern und elektrisch kontrollierbaren Haarhygrometern in 13 Völkern untersucht. Die Rähmchengrößen waren $42 \times 36 \mathrm{~cm}$ und $37 \times 37 \mathrm{~cm}$ (ÖRösI-PÁL, 1932/1935).

Die Temperatur in der Mitte eines gut entwickelten brütenden Bienenvolkes beträgt während der warmen Monate - wie bekannt - von der Außentemperatur unabhängig bei geringer Schwankung $34-35{ }^{\circ} \mathrm{C}$. In den seitlichen Wabengassen und im Honigraum ist die Tempetatur niedriger und schwankt stärker. Die relative Luftfeuchtigkeit ist dagegen in den gleichen Monaten durch große Schwankungen gekennzeichnet. Sie beträgt im Brutraum eines ruhigen Bienenvolkes 30-70\%. Ihre Änderung hängt im großen und ganzen mit den Schwankungen der äußeren Luftfeuchtigkeit zusammen. Die äußere Luftfeuchtigkeit spiegelt sich abgeschwächt in der inneren. Ausserdem beeinflußt die Reifung des Honigs durch Wasserverdunstung stark die Luftfeuchtigkeit innerhalb der Bienenwohnung.

Am Anfang der Wanderung werden die Bienen unruhig. Sie erzeugen so Wärme. Die schädliche Wärme entweicht teilweise durch das Wandergitter oder wird von den Bienen in ihrer Wirkung in zweierlei Weise vermindert : 1. Durch Lockerung der Traube. Die Bienen versuchen hierdurch vor allem die Temperatur des Brutnestes in der Nähe von $35^{\circ} \mathrm{C}$ zu halten. Zu diesem Zweck besetzen sie die kühleren Teile der Bienenwohnung stärker. Dadurch erhöht sich die Temperatur der seitlichen Wabengassen. Aber in einem starken Volk ist eine solche Regulierung oft nicht ausreichend : die Temperatur steigt im Brutraum über $37^{\circ} \mathrm{C}$. Ein “ Trommelraum », d.h. ein belüfteter leerer Raum oben, unten in der Beute oder vor dem Flugloch, wohin sich ein Teil des Volkes zurückzichen kann, hilft in solchen Fällen. - 2. Durch Ausatmen von Wasser. Die wasserhaltige Luft muß durch das Wandergitter entweichen. Die Bienen können den Wasserverlust ihrer Körper ersetzen, indem sie Larven und gedeckelte Brut aussaugen, dabei einen Teil der Brut aus den Zellen herausreißen, oder gedeckelte Honigzellen öffnen, um Honig aufzunehmen.

Junge Bienen sind weniger beweglich. Man wußte das bereits in der Zeit der Korbimkerei, als das Bienenvolk teilweise oder ganz in einen leeren Korb 
( abgetrommelt » wurde. BERLEPSCH (1860) schilderte das mit folgenden Worten : “... das Abtreiben ist gerade zur Zeit des schärfsten Fluges am mißlichsten, weil die jungen Bienen am schlechtesten in die Höhe laufen und die Königin, wenn nicht viele Bienen sie gleichsam mit sich nach aufwärts fortreißen, gern unten sitzen bleibt. ' Man könnte annehmen, daß dies auch während der Wanderung der Fall ist, obschon dann die Erschütterungen viel länger dauern. In Jugoslavien und bei manchen Imkern in Ungarn werden besondere Wanderbeuten nach dem Prinzip von Taмasкo (1956) verwendet. Es wird dabei angenommen, daß während der Wanderung die Jungbienen hauptsächlich im Brutraum bleiben, um die Brut weiter zu pflegen, Trommelräume aber vor allem von älteren Bienen in Anspruch genommen werden.

Meine Versuche seit 1951 bewiesen aber, daß die Brutpflege während der Wanderung sowohl in den Wabenzellen als auch in Weiselzellen stark gestört wird. Versuchsvölker hatten 3 Tage lang Nilblausulfat oder Neutralrot im Futter bekommen, worauf sie einen farbigen Futtersaft erzeugten. Unmittelbar vor der Wanderung gaben wir ihnen Arbeiter- oder Weisellarven mit normalem weißem Futtersaft in Waben oder in künstlichen Weiselzellen. Eine nach der Wanderung unverzüglich durchgeführte Untersuchung verriet, ob die zugegebenen Larven während der Fahrt neuen, farbigen Futtersaft erhalten hatten. Es stellte sich heraus, daß die Bienen während einer 3 Stunden und 30 Minuten bzw. 5 Stunden und 10 Minuten langen Wanderung bei Tage nur einen kleinen Teil der Arbeiter- und Weisellarven gefüttert hatten. Höchstens $1 / 4$ der Arbeiterlarven hatte in dieser Zeit farbiges Futter bekommen (18,7-24,3\%). In einem anderen Versuch wurden ältere Larven gefüttert $(40,5 \%)$, nicht aber 1-2 tägige. Die etwa 2 Tage alten Weisellarven wurden auf der oberen Leiste des Zuchträhmchens (in der Nähe des Trommelraumes) am besten gefüttert, auf der mittleren Leiste weniger, unten gar nicht. Aber sogar oben war die Zahl der gefütterten Larven kleiner und meistens auch die Menge des neuen (farbigen) Futtersaftes geringer als ohne Wanderung (ÖrösI-PÁL, 1965).

Es schien also notwendig, die zahlenmässige Verteilung der Arbeitsbienen nach ihrem Alter im Brutraum und im Trommelraum während der Wanderung festzustellen. Die Versuche wurden in den Jahren 1959, 1960 und 1962 durchgeführt. Ein kurzer Bericht über das Ergebnis wurde in ungarischer Sprache veröffentlicht (ÖnösI-PÁL, 1971).

\section{METHODIK}

Die verwendeten Magazinbeuten hatten im Brutraum je zehn $42 \mathrm{~cm}$ breite und $36 \mathrm{~cm}$ hohe, im Honigraum $42 \mathrm{~cm}$ breite und $13 \mathrm{~cm}$ hohe Rähmchen. Bodenbrett, Brutraum, Honigraum und Trommelraum konnten von einander durch eingeschobene Glasplatten getrennt werden. Es war also möglich, sie nach der Wanderung unabhängig von einander zu untersuchen. Der mit Gitter bedeckte obere Trommelraum war $7 \mathrm{~cm}$ hoch und hatte einen Inhalt von $10,8 \mathrm{dm}^{3}$. 
Breite und Länge entsprachen denen des Brutraumes. Das Flugloch war während der Wanderung luftdicht verschlossen. Das Volk erhielt Luft (und Licht) nur von oben durch vier $25 \times 2 \mathrm{~cm}$ grosse Öffnungen des aufgesetzten flachen Deckels.

Die Volksmenge entsprach jeweils der Jahreszeit. Die Brutmenge wurde mit Hilfe eines auf eine Glasplatte gezeichneten Netzes bestimmt, bei dem jede $18 \times 18 \mathrm{~mm}-$ Einteilung 12 Arbeiterzellen umfaßte.

Die Versuchsbienen ließ ich im Thermostat aus gedeckelter Brut schlüpfen. Sie wurden täglich mit andersfarbigen Flecken auf der Brust gezeichnet und danach von oben in eine Wabengasse des Brutraumes eingelassen. Sie wurden immer gut angenommen. (Durch das Flugloch zugesetzte fremde junge Bienen werden sehr oft angegriffen.) Wenn alle wichtigen Altersklassen der gezeichneten Bienen ausreichend im Volk vertreten waren, begann die Wanderung.

Die Zahlen der gezeichneten Bienen nach der Wanderung entsprachen nie denen der zugesetzten. Im Jahre 1960 blieben von 18819 gezeichneten Bienen nur 760 bis zum Ende der Wanderung übrig. Im Jahre 1962 wurden je Volk 5900 gezeichnete Bienen für den Versuch verwendet, am Ende des Versuches sah ich aber nur 3500 und 2982 je Volk wieder. Der Anteil der übrig gebliebenen gezeichneten Bienen betrug in den 3 Versuchsbeuten 36, 41 und $54,5 \%$. Die Abnahme der Zahl der gezeichneten Bienen zwischen Schlupf und Versuchsbeginn ist bei alten Tieren leicht verständlich. Weitere Ursachen können sein : Verfliegen der Bienen und Abfallen der Farbzeichen. Einige gezeichnete Bienen sah ich vor der Wanderung in anderen Völkern wieder, ein Verfliegen kam also trotz der zahlreichen großen Bäume und Sträucher vor. Die Abnahme der Zahl der Versuchsbienen ist aber nur in den beiden ältesten Klassen mit 29-31 und 35-37 tägigen Bienen so groß, daß die Versuchsergebnisse weniger gesichert erscheinen.

Die Arbeitsteilung im Bienenvolk ist bekanntlich nicht starr. Der Begriff von jungen und alten Bienen hängt nicht immer nur von den Lebenstagen ah, sondern auch vom physiologischen Zustand. Bei meinen Versuchen rechnete ich die 1-14 oder 1-17 Tage alten Bienen zu den jungen.

Beim ersten Versuch (1959) fand ich noch kein richtiges Verfahren für das Zählen der gezeichneten Bienen in den einzelnen Teilen des Versuchsstockes nach der Wanderung. Der Trommelraum wurde für diesen Zweck mit einer seitlich eingeschobenen Glasplatte abgesperrt, abgehoben und in $W$ asser getaucht. Keine Biene konnte wegfliegen. Im Brutraum konnten die Bienen nur mit Wasser besprengt werden. Das Zählen nahm hier mehr Zeit in Anspruch. Ein Teil der gezeichneten Bienen flog inzwischen weg und kam wieder zurück. Bei den Versuchen 1960 und 1962 konnte ich diese Fehler ausschalten. Die Bienen wurden in den einzelnen Beutenteilen von einander getrennt abgeschwefelt und alle Exemplare - auch die ungezeichneten - gezählt.

Ich berücksichtige deshalb hier nur die Versuche von 1960 und 1962.

\section{ERGEBNISSE}

Versuch 1960

Die Bienenmenge des Volkes entsprach der Jahreszeit. Die Brutmenge erreichte 21852 Zellen, was eine tägliche durchschnittliche Eiablage von 1040 bedeutet.

Das Flugloch wurde am 3. August abends geschlossen, 1 Tag nach dem Zusetzen der jüngsten gezeichneten Bienen. Abfahrt am 4. August um $8 \mathrm{Uhr}$ morgens mit einem Lastkraftwagen. Wir fuhren hin und zurück $204 \mathrm{~km}$ in 5 Stunden und 8 Minuten. Unterwegs hielten wir achtmal für 1,5-4 Minuten aus technischen und Verkehrsgründen. Auch bei einer wirklichen Wanderung ist das nicht anders. Die Außentemperatur betrug bei der Abfahrt $18,3^{\circ} \mathrm{C}$, 
um $9 \mathrm{Uhr} 22^{\circ}$, um 11 Uhr $27^{\circ}$, um 12 Uhr $27,8^{\circ}$ und um 13 Uhr $23,8{ }^{\circ} \mathrm{C}$. Nach der Heimkehr wurde das ganze Volk abgeschwefelt und Gewicht und Volumen der Bienen sogleich festgestellt. Das Ergebnis der in den folgenden Tagen vorgenommenen Zählung wird in Tabelle 2 zusammengefaßt.

\section{Versuch 1962}

Es wurden 2 Völker verwendet. Sie waren etwas schwächer als das Volk des Jahres 1960, was sich aber erst bei der Zählung der abgeschwefelten Bienen am Ende des Versuches herausstellte (Tab. 1). Die Brutmenge erreichte 20580 und 22575 Zellen. Dies bedeutet eine tägliche durchschnittliche Eiablage von 980 und 1 075, war also für die Zeit des Versuches normal.

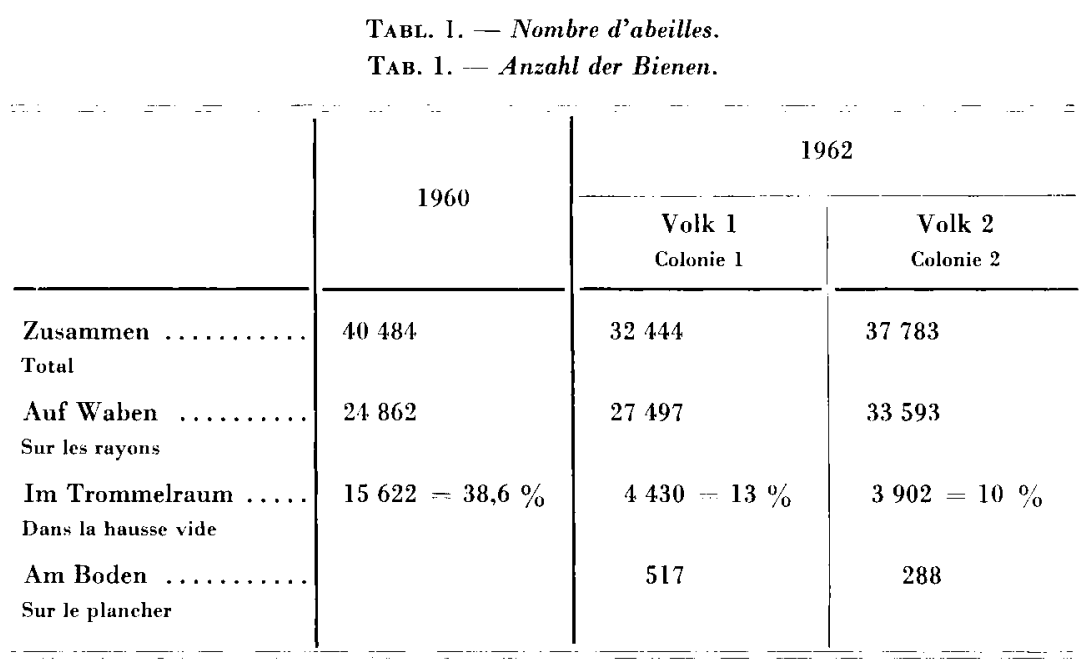

Die Fluglöcher wurden am 11. August abends geschlossen. Abfahrt mit einem Lastkraftwagen am 12. August 8.45 Uhr. Ankunft um 21.38 Uhr. Die Wanderung erstreckte sich auf $244 \mathrm{~km}$. Ungefähr nach $110 \mathrm{~km}$ mußten wir wegen eines Motordefektes von $12.03 \mathrm{Uhr}$ - 15.56 Uhr halten. Die Außentemperatur betrug um 9 Uhr $22^{\circ} \mathrm{C}$, um $9.20 \mathrm{Uhr} 25^{\circ}$, um $10 \mathrm{Uhr} 27,8^{\circ}$, um $11 \mathrm{Uhr} 31,3^{\circ}$, von 12 bis $16 \mathrm{Uhr} 31,8-35,5^{\circ}$, um $17 \mathrm{Uhr} 32^{\circ}$, um $21.30 \mathrm{Uhr}$ $28,8^{\circ} \mathrm{C}$. Die Bienen waren während des Haltens unruhig, sie brausten laut. Durch die Lüftungsöffnungen war zu sehen, daß sie am Wandergitter hin und her liefen und manchmal fliegend an das Gitter stürzten. 


\section{DISKUSSION}

Das größere Volk (1960) hielt den Trommelraum in stärkerem Masse besetzt : 38,6\% der Bienen zogen dorthin (Tab. 1). Bei den etwas kleineren Völkern (1962) haben nur 10-13\% Bienen den Trommelraum in Anspruch genommen (Tab. 1).

Die Verteilung der Bienen nach ihrem Alter in den verschiedenen Beutenteilen fassen die Tabellen 2,3 und 4 zusammen. Im Jahre 1962 wurden auch die Königinnen gefunden und zwar immer im Brutraum.

1962 blieben verhältnismäßig wenige gezeichnete Bienen aus den beiden ältesten Klassen übrig, z. B. im Volk 2 von 2200 nur 55. Im Volk 1 ist die Lage etwas günstiger, aber auch dort ist die Zahl der alten Bienen im Vergleich zu den jungen gering.

Die Tabellen 3 und 4 beweisen trotzdem klar, daß im Jahre 1962 bedeutend weniger junge, für innere Arbeiten geeignete Bienen die Waben verließen, um den Trommelraum in Anspruch zu nehmen. Der Anteil solcher jungen

TAB. 2. - Die Verteilung der gezeichneten Bienen während der Wanderung 1960.

TABL. 2. - Répartition, au cours de la transhumance de 1960, des abeilles marquées.

\begin{tabular}{|c|c|c|c|c|c|}
\hline \multirow{3}{*}{$\begin{array}{c}\text { Alter der } \\
\text { Bienen in Tagen } \\
\text { Age des abeilles } \\
\text { en jours }\end{array}$} & \multirow{3}{*}{$\begin{array}{c}\text { gezeichnet } \\
\text { Anzahl } \\
\text { Nombre } \\
\text { d'abeilles } \\
\text { marquées }\end{array}$} & \multicolumn{4}{|c|}{$\begin{array}{l}\text { Vorgefunden } \\
\text { Abeilles, trouvées à l'arrivée }\end{array}$} \\
\hline & & $\begin{array}{c}\text { zusammen } \\
\text { Anzahl }\end{array}$ & $\begin{array}{c}\text { auf Waben } \\
\text { Anzahl }\end{array}$ & $\begin{array}{l}\text { im Trom } \\
\text { Dans It } h\end{array}$ & $\begin{array}{l}\text { Iraum } \\
\text { se vide }\end{array}$ \\
\hline & & Au total & $\begin{array}{l}\text { sur les } \\
\text { rayons }\end{array}$ & $\begin{array}{l}\text { Anzahl } \\
\text { Nombre }\end{array}$ & $\begin{array}{l}\% \\
\%\end{array}$ \\
\hline $\begin{array}{c}1-2 \\
8-17 \\
20-24 \\
33-42\end{array}$ & $\begin{array}{ll}1 & 480 \\
5 & 050 \\
5 & 230 \\
6 & 959\end{array}$ & $\begin{array}{r}1219 \\
3605 \\
2348 \\
598\end{array}$ & $\begin{array}{r}849 \\
2259 \\
1690 \\
538\end{array}$ & $\begin{array}{r}370 \\
1346 \\
658 \\
60\end{array}$ & $\begin{array}{l}30,3 \\
59,6 \\
28,2 \\
11\end{array}$ \\
\hline $\begin{array}{l}\text { Zusammen } \\
\text { Total }\end{array}$ & 18719 & 7760 & 5336 & 2424 & 38,5 \\
\hline
\end{tabular}

Alter der Bienen im Trommelraum :

Age des abeilles dans la hausse vide :

$1-17$ Tage $\ldots \ldots \ldots \ldots .70,8 \%$

über 17 Tage

$29,2 \%$

1 - 17 jours

plus de 17 jours 
TAB. 3. - Die Verteilung der gezeichneten Bienen während der Wanderung 1962.

ТАвц. 3. - Répartition, au cours de la transhumance de 1962, des abeilles marquées.

Volk 1.

Colonie 1.

\begin{tabular}{|c|c|c|c|c|c|c|}
\hline \multirow{3}{*}{$\begin{array}{c}\text { Alter der } \\
\text { Bienen } \\
\text { in Tagen } \\
\text { Age des abeilles } \\
\text { en jours }\end{array}$} & \multirow{3}{*}{$\begin{array}{c}\text { Gezeichnet } \\
\text { Anzahl } \\
\text { Nombre } \\
\text { d'abeilles } \\
\text { marquées }\end{array}$} & \multirow{3}{*}{$\begin{array}{c}\text { zusammen } \\
\text { Anzahl } \\
\text { Au totul }\end{array}$} & \multicolumn{2}{|c|}{$\begin{array}{c}\text { Vorgefunden } \\
\text { abeilles trouvées à l'arrivée }\end{array}$} & $-\cdots \cdot-$ & \multirow{2}{*}{$\begin{array}{c}\text { am Boden } \\
\begin{array}{c}\text { sur le } \\
\text { plancher }\end{array}\end{array}$} \\
\hline & & & $\begin{array}{c}\text { auf Waben } \\
\text { Anzahl }\end{array}$ & $\begin{array}{rr}\text { im } & \text { Trom } \\
\text { dans la } & \text { la } \\
\end{array}$ & Iraum & \\
\hline & & & sur les rayons & $\begin{array}{l}\text { Anzahl } \\
\text { Nombre }\end{array}$ & $\begin{array}{l}\% \\
\%\end{array}$ & $\begin{array}{l}\text { Anzahl } \\
\text { Nombre }\end{array}$ \\
\hline $\begin{array}{l}1 \\
7-8 \\
13-14 \\
21-23 \\
29-31 \\
35-37\end{array}$ & $\begin{array}{ll} & 600 \\
1 & 000 \\
1 & 000 \\
1 & 100 \\
1 & 200 \\
1 & 000\end{array}$ & $\begin{array}{r}563 \\
919 \\
858 \\
831 \\
282 \\
47\end{array}$ & $\begin{array}{r}545 \\
870 \\
803 \\
708 \\
187 \\
28\end{array}$ & $\begin{array}{r}3 \\
4.6 \\
4.8 \\
115 \\
94 \\
17\end{array}$ & $\begin{array}{l}0,5 \\
5 \\
5,6 \\
13,6 \\
33,8 \\
36\end{array}$ & $\begin{array}{r}15 \\
3 \\
7 \\
8 \\
1 \\
2\end{array}$ \\
\hline $\begin{array}{l}\text { Zusammen } \ldots . \\
\text { Total }\end{array}$ & 5900 & 3500 & 3141 & 323 & 9 & 36 \\
\hline
\end{tabular}

Alter der Bienen im Trommelraum :

Age des abeilles dans la hausse vide

$1-14$ Tage ........ $30 \%$

$1 \cdot 14$ jours

über 14 Tage $\ldots \ldots \ldots 70 \%$ plus de 14 jours

(1-17 und 1-14 Tage alten) Bienen betrug im Trommelraum $1960: 30,3-$ $59,6 \%, 1962: 0,5-15,8 \%$. Hierbei könnte die Tatsache eine Rolle spielen, daß im Jahre 1962 das ganze Volk den Trommelraum überhaupt weniger besetzt hatte.

Man kann aber die Menge der jungen und alten Bienen im Trommelraum besser vergleichen, wenn die Bienen nicht in mehrere kleine, sondern in zwei große Altersgruppen eingeteilt werden : junge Bienen von 1-14 bzw. 1-17 Tagen und alte Bienen darüber. In diesem Sinne waren junge Bienen im Trommelraum in folgenden Prozentzahlen vertreten :

\begin{tabular}{|c|c|}
\hline $1960:$ & $70,8 \%$ \\
\hline 1962 : Volk 1 & 30 \\
\hline Volk 2 & 56 \\
\hline
\end{tabular}


'ГАв. 4. - Die Verteilung der gezeichneten Bienen während der Wanderung 1962.

TABL. 4. - Répartition, au cours de la transhumance de 1962, des abeilles marquêes.

Volk 2.

Colonit 2

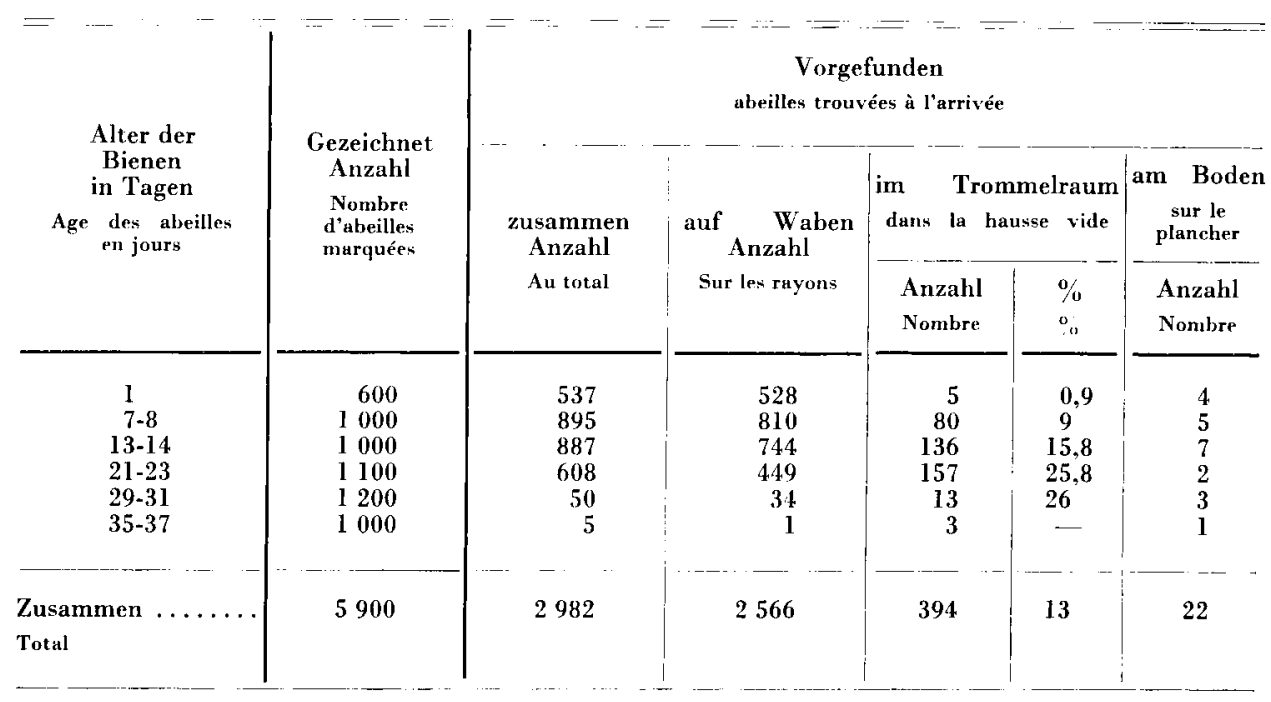

Alter der Bienen im Trommelraum :

Age des abeilles dans la hausse vide :

$1-14$ Tage ........ $56 \%$

1 - 14 jours

über 14 Tage...$\ldots .44 \%$ plus de 14 jours

Die Trennung zwischen alten und jungen Bienen im Brutraum und Trommelraum war 1962 weniger scharf. Es ist wahrscheinlich, daß der Strom nach dem Trommelraum alle Bienen mitreißt, die ihm im Wege stehen, und zwar umso mehr, je größer die Zahl der marschierenden Bienen ist. Das erinnert an das Schwärmen : Auch hier findet man vor dem Flugloch manchmal ganz junge, noch flugunfähige Arbeiterinnen, die von den Schwarmbienen mitgerissen auf der Erde krabbeln.

Ich erachte es damit als bewiesen, daß bei der Wanderung eine bedeutende Menge brutpflegender Bienen den Brutraum verlassen kann. So ist es verständlich, daß bei meinem ersten Versuch mit der farbigen Zuckerlösung nur ein Teil der Larven während der Fahrt gefüttert wurde (ÖrösI-PÁL, 1965). 
Man rechnet auf $1 \mathrm{~kg}$ gewöhnlich 9 000-10 000 Bienen, von Schwarmbienen aber, die mit Honig vollgesogen sind, 7 500-8 000. Ich fand aber 1960, daß die Bienen nach der langen $W$ anderung im Trommelraum leichter waren als die auf den Waben. Im Trommelraum gingen 7266 , auf den Waben nur 6231 Bienen auf $1 \mathrm{~kg}$. Die Erklärung hierfür liegt wohl darin, daß die Bienen auf den Waben während der ganzen Fahrt Honig zu sich nehmen konnten, im Trommelraum hingegen fern von den Honigzellen waren. Nach einer alten Imkerregel soll man den Transport von Bienen nach einigen Minuten für kurze Zeit unterbrechen. Dieser alte Brauch wird jetzt dadurch gerechtfertigt, daß die Bienen so Gelegenheit haben, ihre Honigblasen zu füllen, ehe sie den Trommelraum oder die vom Honig entfernten Teile der Bienenwohnung beziehen.

Eingegangen im Januar 1976.

Reçu pour publication en janvier 1976.

\section{RESUMÉ}

Le transport des ruches rend les abeilles agitées. Pour atténuer les effets nuisibles de la transhumance, on a muni la ruche claustrée d'une grille d'aération et laissé la possibilité aux abeilles de délaisser une partie des rayons pour se regrouper dans un espace vide (hausse vide). Au cours d'une expérience antérieure faite par l'auteur (ÖnösI-PÁL, 1965) on a mis à profit le fait que des abeilles, qui reçoivent du sirop de sucre coloré au rouge neutre ou au sulfate de bleu de Nil, fournissent aux larves de la nourriture larvaire colorée. La couleur de la nourriture larvaire dans les ruches en transhumance prouve que les abeilles ont partiellement négligé les larves d'ouvrières et les larves de reine. Un quart au plus des larves d'ouvrières ont reçu de la nourriture $(18,7-7-24,3 \%)$. Les larves royales situées dans la partie supérieure du cadre d'élevage ont été mieux nourries, celles situées dans la partie médiane, moins bien et celles situées dans la partie inférieure ne l'ont pas été du tout.

Dans une ruche normale, les nourrices sont de jeunes abeilles. Ce résultat ne concorde donc pas avec la constatation selon laquelle de jeunes abeilles ne quittent les rayons de couvain qu'à contrecœur et sans hâte lors d'une secousse (par exemple lorsqu'on chasse les abeilles d'une ruche en paille). Il a paru nécessaire de vérifier la répartition quantitative des ouvrières selon leur âge dans le corps de ruche et la hausse vide au cours de la transhumance.

Les ruches à hausses utilisées pour les expériences étaient équipées de cadres de $42 \times 36 \mathrm{~cm}$ dans le corps et de cadres de $42 \times 13 \mathrm{~cm}$ dans la hausse. La hausse vide, placée tout en haut et recouverte d'un grillage, mesurait $7 \mathrm{~cm}$ de haut et avait une contenance de $10,8 \mathrm{dm}^{3}$. Le trou de vol fut fermé hermétiquement durant la transhumance, la colonie ne recevant air et lumière que par 4 ouvertures latérales pratiquées dans le toit. On fit éclore en étuve, à partir de couvain operculé, les abeilles utilisées pour les expériences. Elles étaient marquées sur le thorax par une pastille de couleur qui variait chaque jour et introduites par le haut entre 2 rayons. On commença la transhumance lorsque toutes les classes d'âge importantes des abeilles marquées furent présentes en nombre suffisant dans la colonie. Les tableaux 2,3 et 4 donnent le nombre d'abeilles marquées. Une partie d'entre elles seulement parvint jusqu'au début de l'expérience (perte du signe coloré), mais il en resta néanmoins suffisamment. Les transhumances en camion eurent toujours lieu au début d'août, de jour et sur une distance comprise entre 204 et $244 \mathrm{~km}$. 
Après la transhumance on sépara aussitôt les divers éléments des ruches par des vitres et on soufra les abeilles. Les jours suivants on compta tous les individus - y compris les non marqués.

\section{Résultats}

Le nombre d'abeilles qui utilisèrent la hausse vide fut très variable : $10-38,6 \%$ de la population totale de la ruche (tabl. 1). Ceci s'explique essentiellement par les fortes différences qui existaient entre les 3 colonies.

Les tableaux 2, 3 et 4 donnent la répartition des abeilles selon leur âge dans les différentes parties des ruches. Il convient de diviser en 2 grands groupes les abeilles qui utilisèrent la hausse vide : les jeunes abeilles (jusqu'à 14 à 17 jours) et les plus vieilles (au-delà de 17 jours). Dans la hausse vide les individus jeunes furent trouvés dans les proportions suivantes : en 1960 : $70,8 \%$; en $1962: 30$ et $56 \%$. Un nombre assez élevé de jeunes abeilles a donc quitté le corps de la ruche pour aller prendre place dans la partie supérieure vide. Ceci explique facilement que, dans une expérience précédente avec du sirop de sucre coloré (Ö́ö̈s-PÁL, 1965), le nourrissement des larves d'ouvrières et de reines ait été insuffisant.

Après une transhumance de $204 \mathrm{~km}$, les abeilles situées dans la hausse vide pesaient moins lourd que celles situées sur les rayons. Il fallait 7266 abeilles prises dans la hausse vide pour faire $1 \mathrm{~kg}$, tandis que 6231 abeilles prises sur les rayons suffisaient; c'est-à-dire que les abeilles de la hausse vide n'avaient pas eu l'occasion de remplir leur jabot de miel au cours du transport. Cette constatation justifie la vieille règle apicole selon laquelle on doit s'arrêter un court moment, quelques minutes après le départ de la transhumance, a fin que les abeilles puissent se gorger de miel.

\section{LITERATUR}

Berlepsch A., 1860. Die Biene und die Bienenzucht. Heinrichshofener Buchhandlung, Mühlhausen. XV, 475 .

Örösi PÁr Z., 1932-1935. A méhcsalád hömérséklete és nedvessége szállitáskor. Mêhêszet, 29-32, Sonderdruck : 1935. Budapest, 1-39. [Ung.].

Ö́ösı PÁL Z., 1965. Die Ernährung der Larven in transportierten Bienenvölkern. Vedecké Práce Vyzkumnych Ustavu Zemedelskych, 137-142.

Örösı PÁL Z., 1971. A szállitott méhek kor szerinti elhelyezkedése. Méhészet, 19, 103-104. [Ung.].

TAмasкo J., 1956. A vándorkaptár szellözéséröl. Méhészet, 4, 63-65. [Ung.]. 